\title{
Erlotinib-induced Adverse Skin Reactions
}

\author{
Toshiyuki Yamamoto*
}

Department of Dermatology, Fukushima Medical University, Fukushima, Japan

\begin{abstract}
Erlotinib is low molecular-weight quinazolin derivatives which selectively inhibit the epidermal growth factor receptor (EGF-R) tyrosine kinase activity of the intracellular domain, block autophosphorylation and the subsequent signaling cascades. EGF-R is expressed on basal keratinocytes, sebocytes, the outer root sheath of hairs, and endothelial cells in the skin, and plays important roles in the regulation of differentiation, proliferation, apoptosis, attachment and migration of keratinocytes, inflammation, and wound healing. Therefore, inhibition of EGF-R causes a number of cutaneous adverse reactions. Among them, severe skin lesions are very stressful, and impair quality of life of patients. Moreover, they even bring disadvantages such as drug withdrawal or interruption. Several review papers describe representative or common skin lesions which appear either during the first a few weeks or at later phases. Common skin manifestations include papular and pustular follicular eruptions (acneiform eruption), xerosis, paronychia, pruritus, and abnormalities of hairs; however, other than those eruptions, several unusual lesions are also induced. Early intervention of dermatologists and management of skin lesions are quite important, because discontinuance of the drug is unfavorable for patients with clinical benefits for cancers. In this brief review, various cutaneous manifestations seen in Japanese patients treated with erlotinib (Tarceva) are shown, and current management of representative severe conditions is also described.
\end{abstract}

Keywords: EGF-R inhibitor, skin, side effect, erlotinib.

\section{INTRODUCTION}

Erlotinib is a novel oral tyrosine kinase inhibitor targeting the epidermal growth factor receptor (EGF-R) and it has been proven to be highly effective in the treatment of chemotherapy-resistant non-small cell lung cancer and pancreatic cancer. In the $75 \%$ of patients treated with erlotinib developed skin reactions, most of which developed within 3 weeks of starting erlotinib [1,2]. The most frequent skin lesion is an acneiform eruption, but xerosis, paronychia, periungual granuloma, pruritus, hair changes, telangiectasia and hyperpigmentation are also known [3-5]. Additionally, uncommon mucocutaneous eruptions such as erosion, nodules, and purpura are also seen. Some of those eruptions appear within a few weeks of treatment onset, while others appear later. In this review, various adverse cutaneous reactions, not only representative but also less frequent conditions, are described which were triggered by erlotinib (Tarceva) in Fukushima Medical University in these 5 years.

\section{PHARMACOLOGY OF ERLOTINIB}

EGF-R is a member of transmembrane receptor tyrosine kinases family, encoded by the c-erb-B proto-oncogene. EGF-R consists of an extracellular ligand-binding receptor domain, a hydrophobic transmembrane domain, and an intracellular tyrosine kinase domain. Erlotinib binds to the ATP-binding site of the tyrosine kinase domain of EGF-R, thereby inhibits the downstream signaling pathways for

*Address correspondence to this author at the Department of Dermatology, Fukushima Medical University, Hikarigaoka 1, Fukushima 960-1295, Japan; Tel; +81 24547 1307; Fax: +81 24547 1307; E-mail: toyamade@fmu.ac.jp cellular proliferation, motility, differentiation, and angiogenesis. In the skin, EGF-R is expressed by basal keratinocytes, sebocytes, the outer root sheath and some endothelial cells and plays a role in the normal differentiation and development of skin follicles and keratinocytes $[6,7]$. EGF$\mathrm{R}$ plays important roles in the regulation of differentiation, proliferation, apoptosis, attachment and migration of keratinocytes, inflammation, and wound healing. EGF-R signals are carefully regulated by inducible feedback inhibitors [8]. Therefore, inhibition of EGF-R causes a number of cutaneous adverse reactions [9]. For example, inhibition of EGF-R downregulates $\mathrm{Ki}-67$ expression in the epidermis, due to reduction of keratinocyte proliferation [10].

\section{SKIN MANIFESTATIONS}

Skin rash is reported to occur in $75 \%$ of patients treated with erlotinib. The most frequently noted eruptions are papulopustular follicular eruptions (acneiform eruption), paronychia, pruritus, dry skin, hair changes, mucositis, and so on. Usually, folliculitis appears after a few (1 to 2) weeks after the initiation of therapies, and exacerbates thereafter [11]. On the other hand, several skin lesions such as paronychia as well as unusual or uncommon manifestations also occur with late onset.

\section{Folliculitis (Acneiform Eruption)}

Folliculitis is the most common side effect (48\% up to $75 \%$ ) exhibiting acneiform eruptions on the face, chest, back, and pubic region (Fig. 1) [12, 13]. Clinical presentations vary such as tiny pustules, inflammatory red papules, erythema with small pustules on the surface, and firm nodules. According to the guidelines of the American National Cancer Institute (Common Terminology Criteria for Adverse 


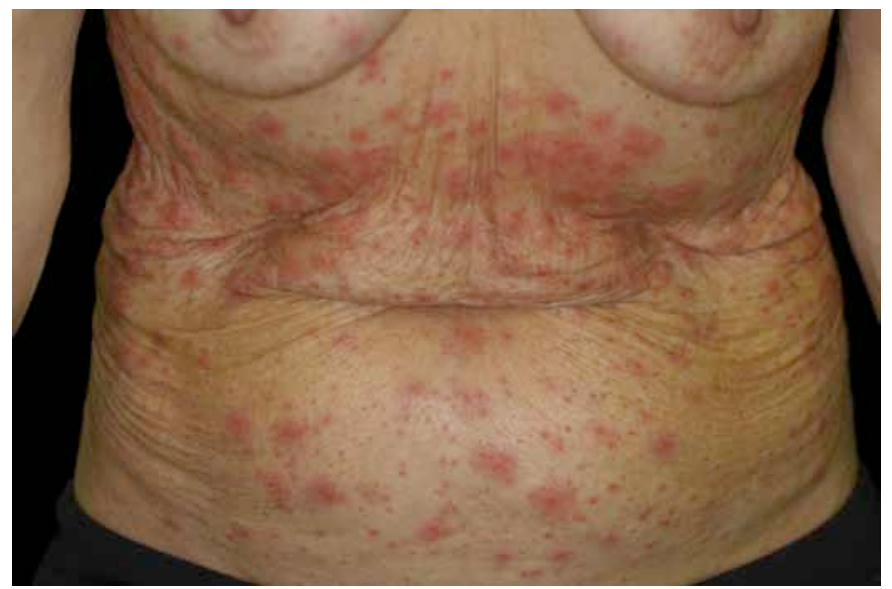

Fig. (1). A number of acneiform eruptions on the trunk.

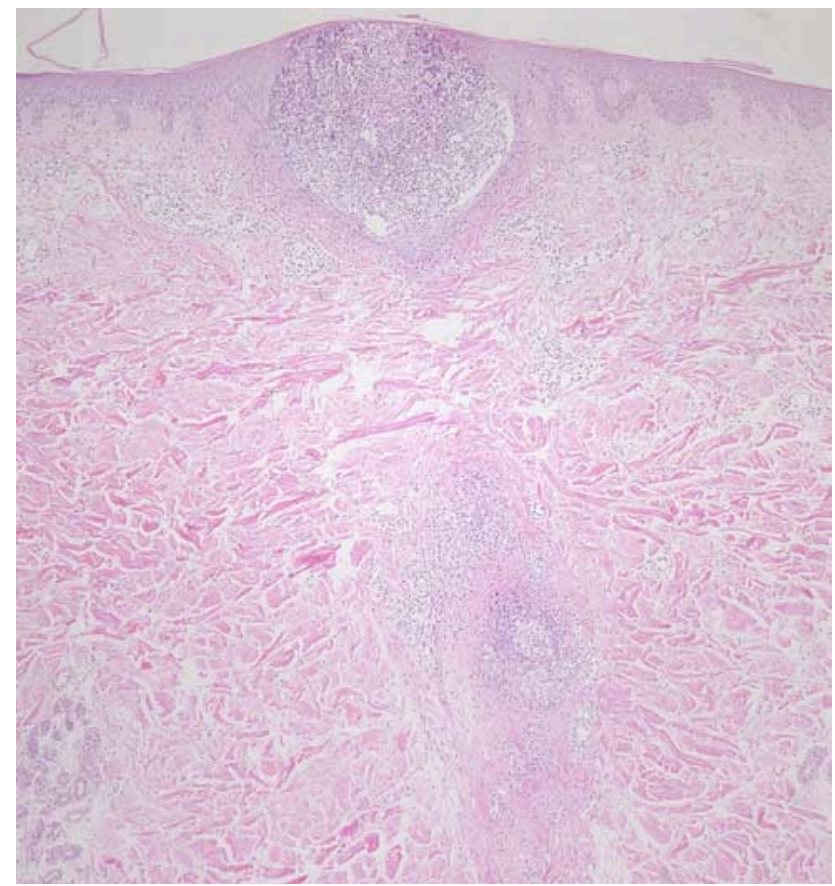

Fig. (2). A biopsy specimen from folliculitis shows intraepidermal neutrophilic abscess and perifollicular inflammation in the dermis.

Events (CTCAE)), rash is classified into Grade 1 (papules and/or pustules covering $<10 \%$ of body surface areas, which may or may not be associated with symptoms of pruritus or tenderness), Grade 2 (papules and/or pustules covering 10$30 \%$ body surface areas, which may or may not be associated with symptoms of pruritus or tenderness; associated with psychosocial impact; limiting instrumental ADL), Grade 3 (papules and/or pustules covering $>30 \%$ body surface areas, which may or may not be associated with symptoms of pruritus or tenderness; limiting self care ADL; associated with local superinfection with oral antibiotics indicated), Grade 4 (papules and/or pustules covering any \% body surface areas, which may or may not be associated with symptoms of pruritus or tenderness and are associated with extensive superinfection with IV antibiotics indicated; lifethreatening consequences), and Grade 5 (death). In general, rash development classified to grade 2 or higher grade is suggested to be a favorable reaction to highly respond with high clinical benefits and show prolonged survival duration [14-16]. On the other hand, Multinational Association of
Supportive Care in Cancer (MASCC) grading for papulopustular eruption adopts the objective measures (number of papulopustules, areas of erythema or edema $<1 \mathrm{~cm}$ in size), as well as health-related quality of life.

Erlotinib acts through competitive binding of the adenosine triphosphate-binding domain of the receptor, and inhibits the proliferation of various cancer cells. EGF-R is expressed in the basal layer of the epidermis, outer root sheath of the hair follicles, and sebaceous and sweat gland apparatus. Therefore, inhibition of EGF-R may result in abnormal epidermal differentiation leading to follicular obstruction and subsequent inflammation. Anceiform eruptions spare the previously irradiated skin, where hair follicles and sebaceous glands are destructed $[17,18]$. Although EGF-R usually does not exert effects on sebocytes [10], further studies are necessary. Biopsy specimens show cellular infiltrates containing neutrophils around the hair follicles, and also intraepidermal neutroplilic abscess formation (Fig. 2), which are preceded by T cell infiltration surrounding the follicular infundibulum [7]. 


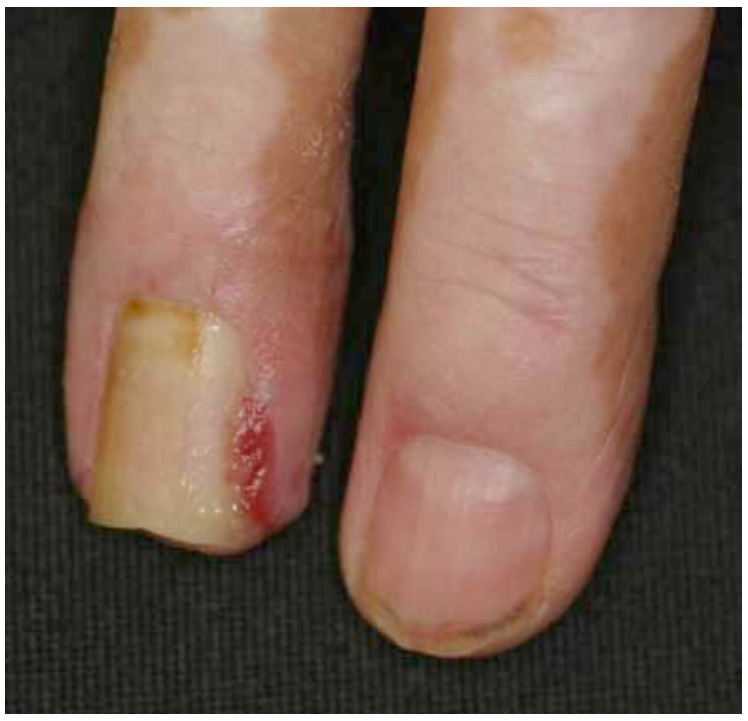

Fig. (3). Paronychial inflammation and pyogenic granuloma on the fingernail.

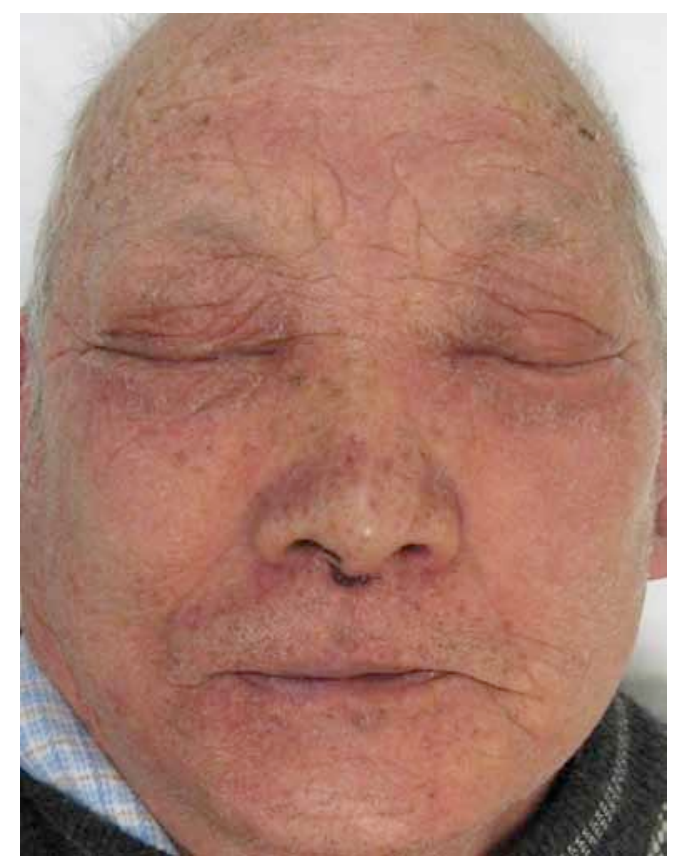

Fig. (4). Scaly erythema on the forehead, around the eyes, and nasolabial areas, along with folliculitis.

Therapies are usually performed by topical clindamycin, metronidazole, nadifloxacin, and adapalene gel. In more severe cases, topical corticosteroids are not effective, and oral tetracyclines (minocycline or doxycycline) are used. Some patients present with severe follicular eruption, and are forced to stop the drug temporally. Decrease of the drug, unless discontinuance, is the effective approach for relieving symptoms; however, folliculitis may deteriorate repetitively during the course. To date, several studies have been reported on the curative and preventive/prophylactic management of EGF-R inhibitor-induced folliculitis [19]. Although not randomized and evidence level is low, curative studies have shown that oral antibiotics are effective.

Administration of doxycycline $(100 \mathrm{mg} / \mathrm{day})$ resulted in a preventive effect for patients receiving elrotinib with grade
$2-3$ severity, compared with placebo (39\% versus $80 \%$ ) at 4 months [19]. Additionally, including various kinds of EGF-R inhibitors (erlotinib, cetuximab, panitumumab, gefitinib), several studies have examined the preventive effects of doxycycline $(100 \mathrm{mg})$ or minocycline $(100 \mathrm{mg})$ for $6-8$ weeks, as well as topical hydrocortisone [20-23], some of which failed to show a statistically significant effect of tetracycline (500 mg twice a day for 4 weeks), as compared with placebo $[20,21]$. In a STEPP trial including patients receiving panitumumab with grade $2-3$, doxycycline (100 mg twice a day) reduced the incidence of grade 2 to 3 toxicity as compared with placebo (29\% versus $62 \%)$ at the endopoint of 6 weeks [23]. Another study for patients receiving cetuximab showed that minocycline $(100 \mathrm{mg} / \mathrm{day})$ resulted in a favorable response at 4 weeks, rather than at 8 weeks. Steroid-induced prominent telangiectasia is sometimes seen on the face. In a prospective randomized trial, half-side test of proactive topical pimecrolimus treatment showed greater decrease in lesion numbers by the investigators' evaluation at weeks 2 and 5, whereas not significant by patients' assessment [24]. Overall, for patients with grade 2 or higher, topical hydrocortisone $1 \%$ cream combined with moisturizer and sunscreen, and oral minocycline $(100 \mathrm{mg} /$ day $)$ or doxycycline $(100 \mathrm{mg} /$ day $)$ are recommended as a preventive therapy, and topical alclometasone $0.05 \%$ cream, fluocinonide $0.05 \%$ cream, or clyndamycin $1 \%$, and oral doxycycline (100 $\mathrm{mg}$ /day), minocycline (100 mg/day) or isotretinoin (20-30 $\mathrm{mg}$ /day) are recommended for curative therapy [25].

\section{Paronychia}

Paronychia appears on the fingers and toes 1 to 3 months of erlotinib therapy, which occurs as painful erythema and occasionally develops pyogenic granuloma-like lesions (Fig. 3). Periungual redness and abscesses are observed in $40-50 \%$ of patients $[26,27]$. Those changes are either triggered by nail-cut and minor trauma, or occur spontaneously. Bacterial infections develop at the nail lesions [28], which may be secondary, not primary event. Various therapies similar to ingrown nail are used, such as biotin intake, topical antibiotics, topical corticosteroids, silver nitrate, cryotherapy, electrocautery, and taping. Tetracycline is sometimes effective, which may be by inhibiting inflammation and reactive oxygen species, in addition to its antibiotic efficacy.

\section{Seborrheic Dermatitis-like Eruption}

Seborrheic dermatitis-like scaly erythema are observed on the face and trunk (Fig. 4), which may be intermingled with acneiform eruptions or xerotic eczema. Topical corticosteroids, calcineurin inhibitors, anti-fungal ointment, and metronidazole gel are used.

\section{Xerosis}

Dry skin with thin scales or pityriasis on the surface is frequently observed on the back and extremities in patients treated with erlotinib (Fig. 5), with ratios of 12 to $35 \%$ in clinical trials [16]. Xerotic skin develops after 1 to 2 months from onset of erlotinib therapy. In vitro studies have shown that inhibition of EGF-R causes arrest of keratinocyte growth and initiates terminal differentiation [29], which may explain the clinical symptoms of xerosis. Patients are encouraged to moist their body by emollient creams. 


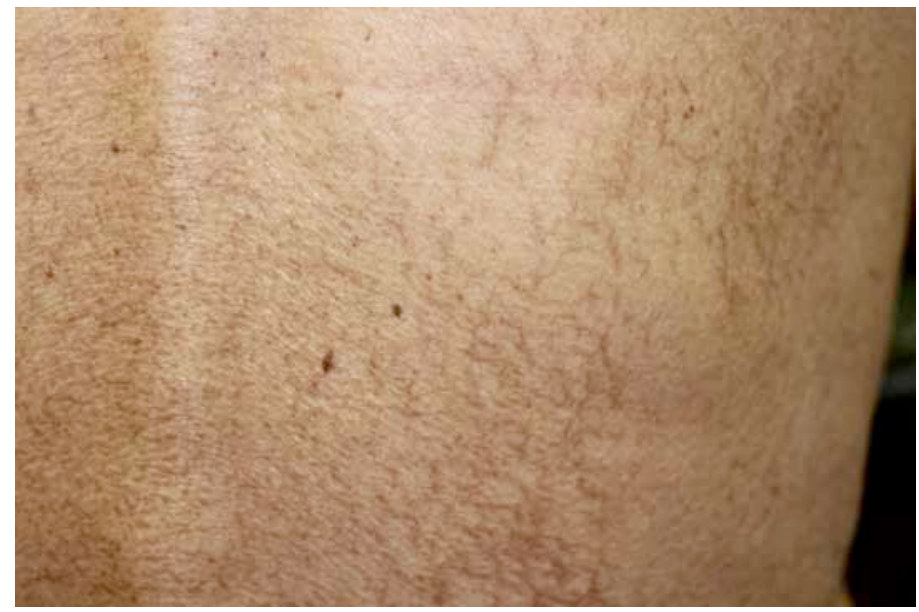

Fig. (5). Xerosis with rippled-pattern scales on the back.

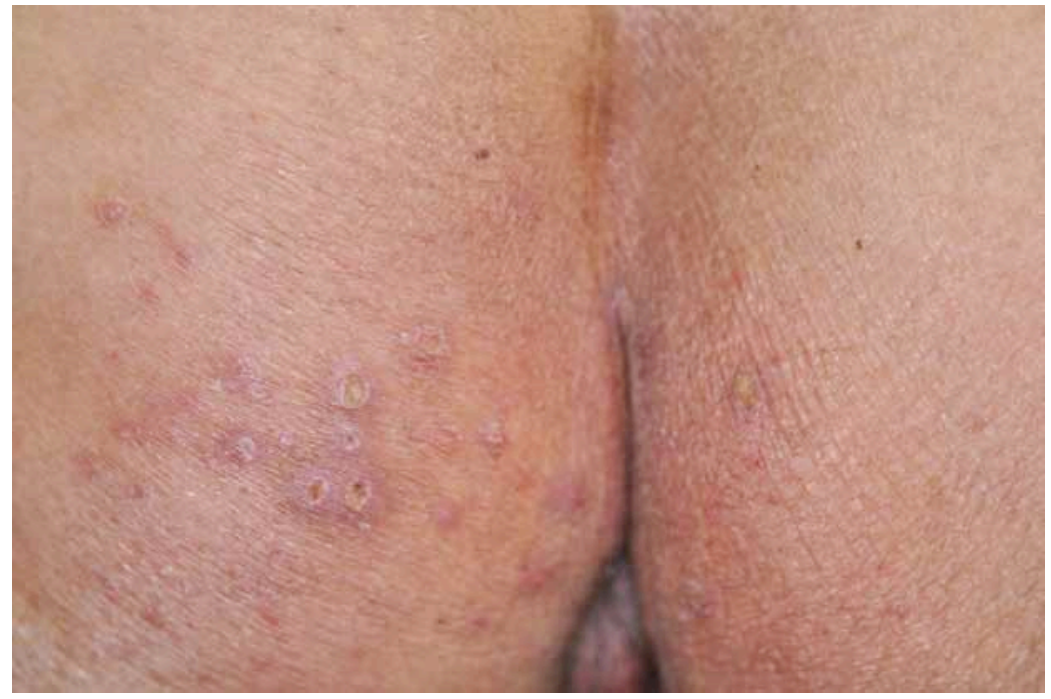

Fig. (6). Prurigo nodules on the buttocks.

\section{Pruriginous Reaction}

Prurigo-like lesions are sometimes seen on the lower extremities or buttocks (Fig. 6). Pruriginous eruption may be induced by scratching because of pruritus. Also, occurrence of acquired reactive perforating collagenosis (ARPC) is reported which was simultaneously developed with follicular eruption and paronychia [30]. ARPC is histologically characterized by necrobiotic basophilic collagen bundles which run transversely through the epidermis. Clinically, a number of umbilicated papules and nodules with central keratotic plugs develop on the trunk and extremities. ARPC is triggered by minor trauma, such as folliculitis, arthropod bites, and scabies infection. Koebnerization seems to play an important role. Scratching of patients suffering from pruritus may cause microtrauma and necrobiosis of the dermal structure. Insufficient blood supply as a result of vasculopathy may be associated as the underlying factor in ARPC. Follicular occlusion caused by altered differentiation of the epithelium due to inhibition of EGF-R may induce ARPC.

\section{Eyelash Trichomegaly}

Prolonged, curly eyelashes are sometimes seen after 7 weeks to 5 months, or other studies show mean duration of
173 days (Fig. 7). Trichomegaly is caused by premature maturation of the hair of the eyelashes. Prolonged eyebrow, curly hairs, and even the darkening of the hair may be simultaneously seen in a single individual [31]. EGF-R plays a role in the regulation of hair growth cycles, as a switch to begin and end the anagen phase of the hair cycle [32]. It is also suggested that hair growth speed is different depending on different location, such as hair, cheek, ear, eye, and trunk.
Alopecia
Alopecia has been reported in $5 \%$ of patients treated with EGF-R inhibitors after several months [9]. Abscess forma- tion due to folliculitis on the scalp is occasionally seen (Fig. 8), which later results in, either non-scarring or scarring, alopecia. Histological examination of the scalp alopecia of patients treated with EGF-R inhibitors shows marked in- flammatory infiltrate that consists of lymphocytes, plasma cells and some neutrophils and eosinophils [18]. Scarring alopecia showing an increased number of telogen and catagen hairs with perifollicular chronic inflammation and perifollicular fibrosis is also reported [33]. In vitro models have shown that the activation of the EGF-R is involved in triggering the transition from anagen to catagen [34]. EGF-R is appeared to be an important mediator in order to enter into 


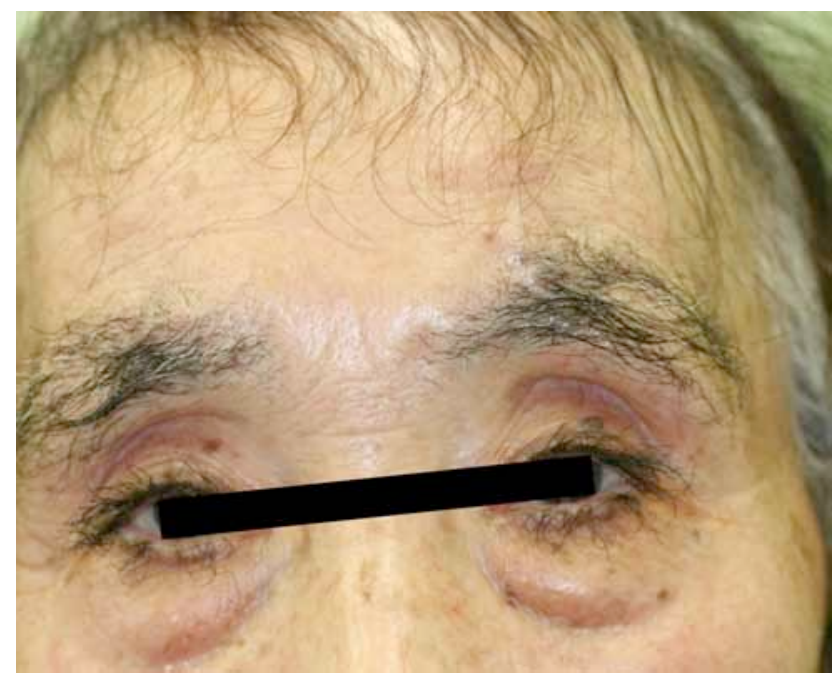

Fig. (7). Prolonged, curly eyelashes and eyebrows. Note that scalp hairs also show curling.

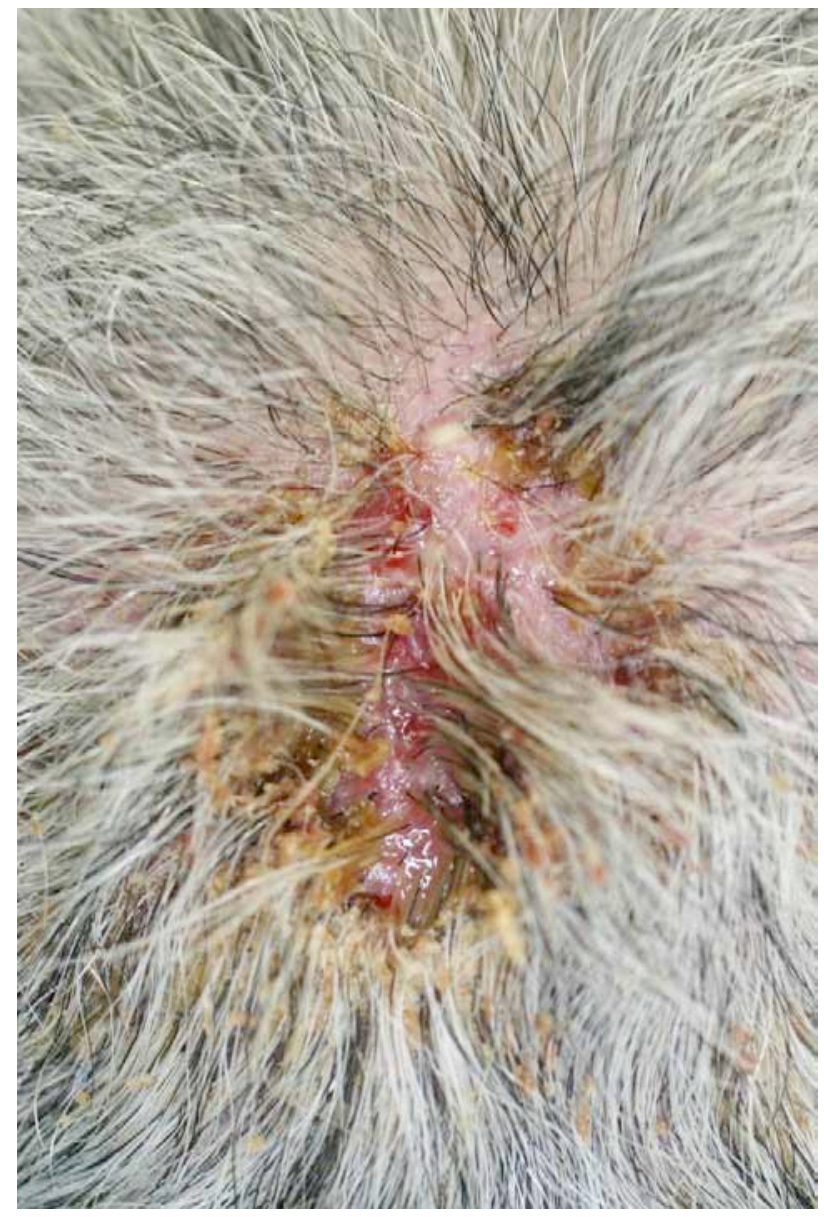

Fig. (8). Abscess with deep ulcers on the scalp.

the catagen stage for the regulation of hair cycle. Inhibition of EGF-R may result in the corruption of regulatory hair cycle and increased production of inflammatory mediators. EGF-R null mice exhibit alopecia [35], and skin graft from EGF-R null mice onto nude mice that express EGF-R shows follicles with an abundant inflammation and leads to alopecia by 10 weeks [36]. Frontal alopecia resembling malepattern baldness is also seen [3, 37]. This type of hair loss can result from severe abscess on the scalp. Another possi- bility may be that EGF-R modifies androgen hormonal signaling. Topical corticosteroids, minoxidil, and oral tetracycline for causative conditions such as severe folliculitis, are used.

\section{Purpura}

Purpuric drug eruption due to the EGF-R inhibitors is a rare skin reaction, which appear at later stages. So far, only few cases of purpuric eruption associated with erlotinib have 


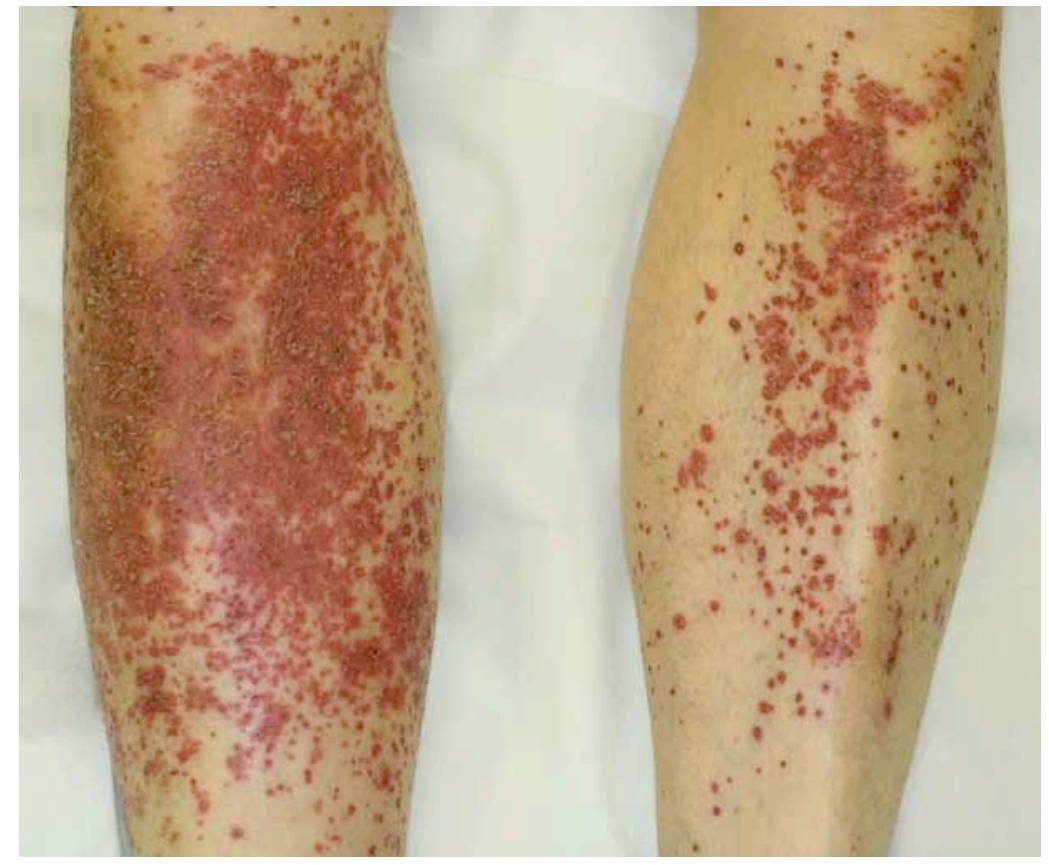

Fig. (9). Severe purpuric plaques on the bilateral lower legs. Xerosis is also seen.

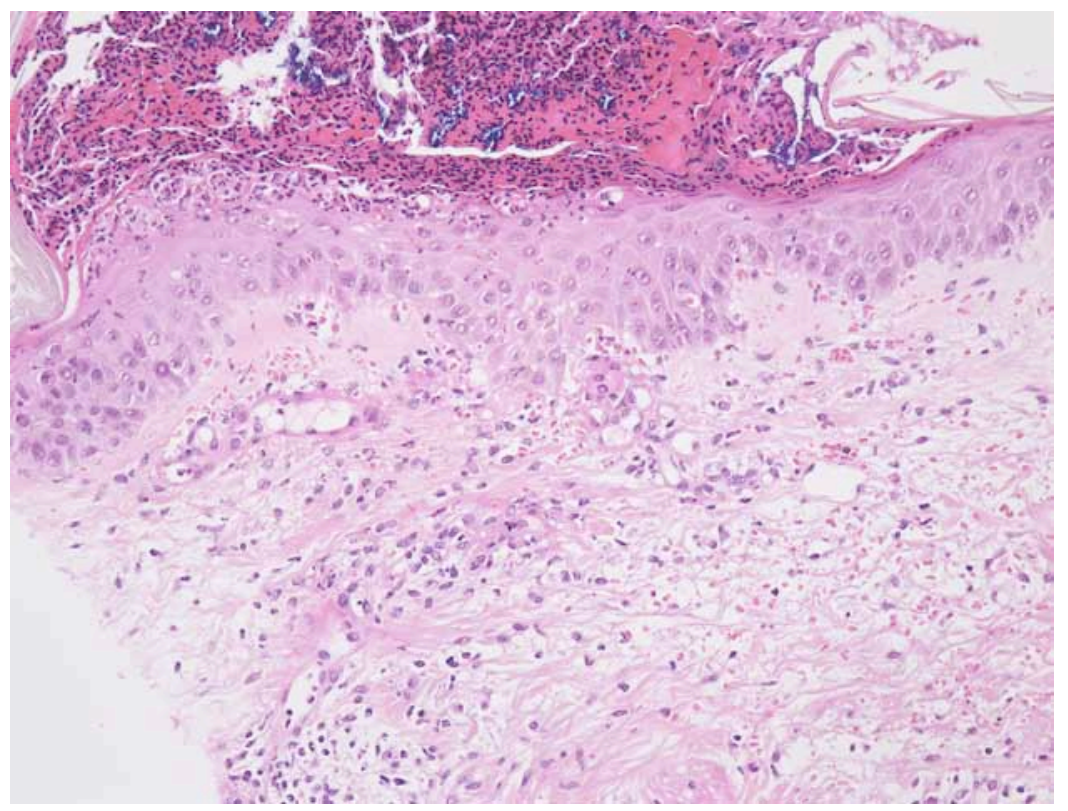

Fig. (10). Histological features of purpuric lesions showing subcorneal neutrophil infiltration, perivascular infiltration of inflammatory cells, fibrin deposition, and extravasation of red blood cells in the upper dermis.

been reported [37]. Purupura is most frequently seen on the lower extremities, and often associated with xerosis or folliculitis. Purpuras vary from pin-point tiny purpuras to severe purpuras coalescing large plaques mimicking HenochSchönline purpura (Fig. 9). Purpuric lesions are also seen on the trunk, intermingled with folliculitis and xerotic scales. Histologically, prominent extravasation of red blood cells, fibrin deposition in the blood vessels, and cellular infiltrates of lymphocytes and neutrophils are seen in the upper dermis (Fig. 10), but features of leukocytoclastic vasculitis are not observed. Inhibition of EGF-R on the endothelial cells of cutaneous vessels may contribute to induce minor vascular impairment and extravasation of erythrocytes, leading to purpuric eruption. On the other hand, EGF-R targeted ther- apy affect the immune response with increased inflammatory cell chemoattractants. Perivascular infiltrations lead to vessel wall damage that results in ecstatic vessels and extravasation of erythrocytes [9]. Long after the condition of dermal congestion and platelet aggregation, extravasation may be induced and purpuric reaction occur eventually. Topical corticosteroids are usually chosen, and in cases of severe purpuric eruptions, dose-reduction or temporal withdrawal is needed.

\section{Other Rare Skin Lesions}

Painful aphthae appear on the oral mucosa, lips, and tongue (Fig. 11). If they increased in number, sometimes they disturb patients' quality of life. Superficial erosive lesions are observed on the buttock (Fig. 12). Also, painful 


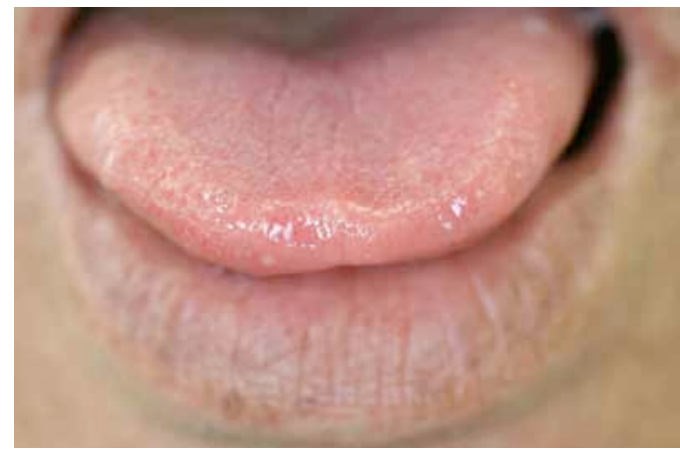

Fig. (11). Aphtha on the tip of the tongue.

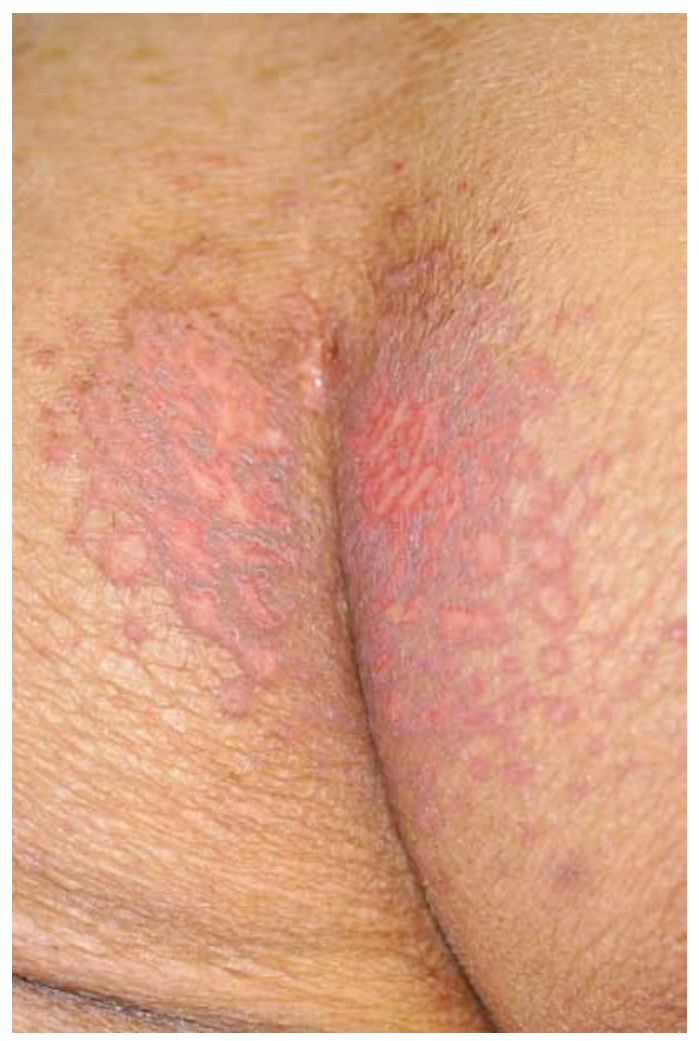

Fig. (12). Erosions and superficial ulcers on the buttock.

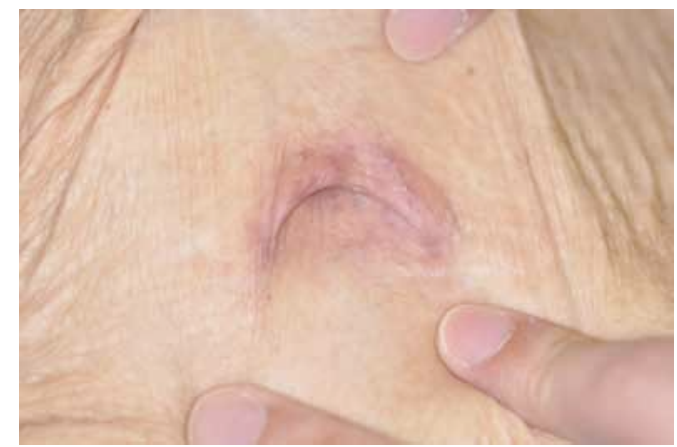

Fig. (13). Erythema exclusively involving the umbilicus.

erosions may appear on the genital or anal regions. Eczematous eruptions with pruritus are often seen. Erythema on the umbilicus are rarely seen (Fig. 13), which may be included in seborrheic dermatitis. Otherwise, photosensitivity is reported in some patients, and development of xanthoma on the sun-exposed areas is also shown [38]. A recent study has suggested a correlation between skin phototype and rash severity, and lower skin phototype may induce severe grade rash [39]. Furthermore, acute generalized exanthematous pustulosis characterized by severe generalized aseptic pustules has been reported [40].

\section{CONCLUSION}

Because the cutaneous toxicity is dose-related, the most effective way for the management of skin rash is the interruption of erlotinib. However, since the occurrence of skin rash is associated with the response rates and survival duration [1, 41], discontinuance of the drug is unbeneficial for the treatment of cancers. Thus, role of dermatologists is important to control skin rash tolerated, as long as the rash does not reach grade 3 (severe or medically significant but not immediately lifethreatening; hospitalization or prolongation of hospitalization indicated; disabling; limiting self care ADL). Manuals of the management of skin lesions by expert opinion have been proposed, i.e. proactive treatments, or dose-escalation trial, depending on the severity grades, from different regions [42-48]. However, randomized control studies are still few, and for only limited and frequently seen conditions. We need to explore more effective treatments for cutaneous lesions, and further studies are necessary to investigate which type of skin eruption is the most relevant surrogate marker for clinical prognosis, or to compare the responders and non-responders among patients with cutaneous toxicities.

\section{CONFLICTS OF INTEREST}

The author confirms that this article content has no conflicts of interest.

\section{ACKNOWLEDGEMENTS}

Declared none.

\section{REFERENCES}

[1] Soulieres D, Senzer NN, Vokes EE, et al. Multicenter phase II study of erlotinib, an oral epidermal growth factor receptor tyrosine kinase inhibitor, in patients with recurrent or metastatic squamous cell cancer of the head and neck. J Clin Oncol 2004; 22: 77-85.

[2] Shepherd FA, Rodrigues Pereira J, Ciuleanu T, et al. Erlotinib in previously treated non-small-cell lung cancer. N Engl J Med 2005; 353: 123-32.

[3] Robert C, Soria J-C, Spatz A, et al. Cutaneous side-effects of kinase inhibitors and blocking antibodies. Lancet Oncol 2005; 6: 491-500.

[4] Heidary N, Naik H, Burgin S. Chemotherapeutic agents and the skin: an update. J Am Acad Dermatol 2008; 58: 545-70.

[5] Hu JC, Sadeghi P, Pinter-Brown LC, et al. Cutaneous side effects of epidermal growth factor receptor inhibitors: clinical presentation, pathogenesis, and management. J Am Acad Dermatol 2007; 56: 317-26.

[6] Busam KJ, Capodieci P, Motzer R, et al. Cutaneous side-effects in cancer patients treated with the antiepidermal growth factor receptor antibody C225. Br J Dermatol 2001; 144: 1169-76.

[7] Nanney LB, Magid M, Stoscheck CM, et al. Comparison of epidermal growth factor binding and receptor distribution in normal human epidermis and epidermal appendages. J Invest Dermatol 1984; 83: 385-93.

[8] Segatto O, Anastasi S, Alema S. Regulation of epidermal growth factor receptor signaling by inducible feedback inhibitors. J Cell Sci 2011; 124: 1785-93.

[9] Lcouture ME. Mechanisms of cutaneous toxicities to EGFR inhibitors. Nat Rev Cancer 2006; 6: 803-12. 
[10] Albanell J, Rojo F, Averbuch S, et al. Pharmacodynamic studies of the epidermal growth factor receptor inhibitor ZD1839 in skin from cancer patients: histopathologic and molecular consequences of receptor inhibition. J Clin Oncol 2002; 20: 110-24.

[11] Boone SL, Rademaker A, Liu D, et al. Impact and management of skin toxicity associated with anti-epidermal growth factor receptor therapy: survey results. Oncology 2007; 72: 152-9.

[12] Cohen EE, Rosen F, Stadler WM, et al. Phase II trial of ZD1839 in recurrent or metastatic squamous cell carcinoma of the head and neck. J Clin Oncol 2003; 21: 1980-7.

[13] Perez-Soler R. Can rash associated with HER1/EGFR inhibition be used as a marker of treatment outcome? Oncology (Williston Park) 2003; 17 (11Suppl 12): 23-8.

[14] Wacker B, Nagrani T, Weinberg J, et al. Correlation between development of rash and efficacy in patients treated with the epidermal growth factor receptor tyrosine kinase inhibitor erlotinib in two large phase III studies. Clin Cancer Res 2007; 13: 3913-21.

[15] Pérez-Soler R, Chachoua A, Hammond LA, et al. Determinants of tumor response and survival with erlotinib in patients with nonsmall-cell lung cancer. J Clin Oncol 2004; 22: 3238-47.

[16] Tsimboukis S, Merikas I, Karapanagiotou EM, et al. Erlotinibinduced skin rash in patients with non-small-cell lung cancer: pathogenesis, clinical significance, and management. Clin Lung Cancer 2009; 10: 106-11.

[17] Mitra SS, Simcock R. Erlotinib induced skin rash spares skin in previous radiotherapy field. J Clin Oncol 2006; 24: e28-9.

[18] Acharya J, Lyon C, Bottomley DM. Folliculitis-perifolliculitis related to erlotinib therapy spares previously irradiated skin. J Am Acad Dermatol 2009; 60: 154-7.

[19] Bachet J-B, Peuvrel L, Bachmeyer C, et al. Folliculitis induced by EGFR inhibitors, preventive and curative efficacy of tetracyclines in the management and incidence rates according to the type of EGFR inhibitor administered: a systematic literature review. Oncologist 2012; 17: 555-68.

[20] Jatoi A, Rowland K, Sloan JA, et al. Tetracycline to prevent epidermal growth factor receptor inhibitor-induced skin rashes: results of a placebo-controlled trial from the North Central Cancer Treatment Group (N03CB). Cancer 2008; 113: 847-53.

[21] Jatoi A, Dakhil SR, Sloan JA, et al. Prophylactic tetracycline does not diminish the severity of epidermal growth factor receptor (EGFR) inhibitor-induced rash: results from the North Central Cancer Treatment Group (Supplementary N03CB). Support Care Cancer 2011; 19: 1601-7.

[22] Scope A, Agero AL, Dusza SW, et al. Randomized double-blind trial of prophylactic oral minocycline and topical tazarotene for cetuximab-associated acne-like eruption. J Clin Oncol 2007; 25: 5390-6.

[23] Lacouture ME, Mitchell EP, Piperdi B, et al. Skin toxicity evaluation protocol with panitumumab (STEPP), a phase II, open-label, randomized trial evaluating the impact of a pre-emptive skin treatment regimen on skin toxicities and quality of life in patients with metastatic colorectal cancer. J Clin Oncol 2010; 28: 1351-7.

[24] Scope A, Lieb JA, Dusza SW, et al. A prospective randomized trial of topical pimecrolimus for cetuximab-associated acne-like eruption. J Am Acad Dermatol 2009; 61: 614-20.

[25] Lacouture ME, Anadkat MJ, Bensadoun R-J, et al. Clinical practice guidelines for the prevention and treatment of EGFR inhibitorassociated dermatologic toxicities. Support Care Cancer 2011; 19: 1079-95.

[26] Osio A, Mateurs C, Soria JC, et al. Cutaneous side-effects in patients on long-term treatment with epidermal growth factor receptor inhibitors. Br J Dermatol 2009; 161: 515-21.

[27] Roé E, Garcia Muret MP, Marcuello E, et al. Description and management of cutaneous side effects during cetuximab or erlotinib treatments: a prospective study of 30 patients. J Am Acad Dermatol 2006; 55: 429-37.
[28] Eilers Jr RE, Gandhi M, Patel JD, et al. Dermatologic infections in cancer patients treated with epidermal growth factor receptor inhibitor therapy. J Natl Cancer Inst 2010; 102: 47-53.

[29] Peus D, Hamacher L, Pittelkow MR. EGF-receptor tyrosine kinase inhibition induces keratinocyte growth arrest and terminal differentiation. J Invest Dermatol 1997; 109: 751-6.

[30] Suzuki Y, Yamamoto T. Reactive perforating collagenosis during erlotinib therapy. Acta Derm Venereol 2012; 92: 216-7.

[31] Alexandrescu DT, Kauffman CL, Dasanu CA. Persistent hair growth during treatment with the EGFR inhibitor erlotinib. Dermatol Online J 2009; 15: 4.

[32] Mak KK, Chan SY. Epidermal growth factor as a biologic switch in hair growth cycle. J Biol Chem 2003; 278: 26120-6.

[33] Hepper DM, Wu P, Anadkat MJ. Scarring alopecia associated with the epidermal growth factor receptor inhibitor erlotinib. J Am Acad Dermatol 2011; 64; 996-7.

[34] Philpott MP, Kealey T. Effect of EGF on the morphology and patterns of DNA synthesis in isolated human hair follicules. J Invest Dermatol 1994; 102; 186-91.

[35] Murillass R, Larcher F, Conti CJ, et al. Expression of dominant negative mutant of epidermal growth factor receptor in the epidermis of transgenic mice elicits striking alterations in hair follicle development and skin structure. EMBO J 1995; 14; 5216-23.

[36] Hansen LA, Alexander N, Hogan ME, et al. Genetically null mice reveal a central role for epidermal growth factor receptor in the differentiation of the hair follicle and normal hair development. Am J Pathol 1997; 150: 1959-75.

[37] Nakamura-Wakatsuki T, Yamamoto T. Purpuric drug eruption and alopecia induced by erlotinib. Dermatol Online J 2012; 18: 16.

[38] Eames T, Kroth J, Flaig MJ, et al. Perifollicular xanthomas associated with epidermal growth factor receptor inhibitor therapy. Acta Derm Venereol 2010; 90: 202-3.

[39] Luu M, Boone SL, Patel J, et al. Higher severity grade of erlotinibinduced rash is associated with lower skin phototype. Clin Exp Dermatol 2011; 36: 733-8.

[40] Liquete E, Ali S, Kammo R, et al. Acute generalized exanthematous pustulosis induced by erlotinib (Tarceva) with superimposed Staphylococcus aureus skin infection in a pancreatic cancer patient: a case report. Case Rep Oncol 2012; 5: 253-9.

[41] Jackman DM, Yeap BY, Lindeman NI, et al. Phase II clinical trial of chemotherapy-naïve patients $>70$ years og age treated with erlotinib for advanced non-small-cell lung cancer. J Clin Oncol 2007; 29: 760-6.

[42] Lacouture ME, Melosky BL. Cutaneous reactions to anticancer agents targeting the epidermal growth factor receptor: a dermatology-oncology perspective. Skin Therapy Lett 2007; 12: 1-9.

[43] Eaby B, Culkin A, Lacouture ME. An interdisciplinary consensus on managing skin reactions associated with human epidermal growth factor receptor inhibitors. Clin J Oncol Nurs 2008; 12: 28390.

[44] Potthoff K, Hofheinz R, Hassel JC, et al. Interdisciplinary management of EGFR-inhibitor-induced skin reactions: a German expert opinion. Ann Oncol 2011; 22: 524-35.

[45] Thatcher N, Nicolson M, Groves RW, et al. Expert consensus on the management of erlotinib-associated cutaneous toxicity in the U.K. Oncologist 2009; 14: 840-7.

[46] Melosky B, Burkes R, Rayson D, et al. Management of skin rash during EGFR-targeted monoclonal antibody treatment for gastrointestinal malignancies: Canadian recommendations. Curr Oncol 2009; 16: 16-26.

[47] Lemmens L. Management of dermatologic toxicities related to epidermal growth factor receptor inhibitor therapy across Europe: can we get a consensus? Ecancermedicalscience 2011; 5: 220.

[48] Kiyohara Y, Yamazaki N, Kishi A. Erlotinib-related skin toxicities: treatment strategies in patients with metastatic non-small cell lung cancer. J Am Acad Dermatol 2013; 69: 463-72. 